\title{
NONCOMMUTATIVE $L$-FUNCTIONS FOR VARIETIES OVER FINITE FIELDS
}

\author{
MALTE WITTE
}

\begin{abstract}
In this article we prove a Grothendieck trace formula for $L$-functions of not necessarily commutative adic sheaves.
\end{abstract}

\section{INTRODUCTION}

Let $\mathcal{F}$ be an $\ell$-adic sheaf on a separated scheme $X$ over a finite field $\mathbb{F}$ of characteristic different from $\ell$. The $L$-function of $\mathcal{F}$ is defined as the product over all closed points $x$ of $X$ of the characteristic polynomials of the geometric Frobenius automorphism $\mathfrak{F}_{x}$ at $x$ acting on the stalk $\mathcal{F}_{x}$ :

$$
L(X, \mathcal{F}, T)=\prod_{x} \operatorname{det}\left(1-\mathfrak{F}_{x} T^{\operatorname{deg} x}: \mathcal{F}_{x}\right)^{-1} .
$$

The Grothendieck trace formula relates the $L$-function to the action of the geometric Frobenius $\mathfrak{F}_{\mathbb{F}}$ on the $\ell$-adic cohomology groups with proper support over the base change $\bar{X}$ of $X$ to the algebraic closure:

$$
L(X, \mathcal{F}, T)=\prod_{i \in \mathbb{Z}} \operatorname{det}\left(1-\mathfrak{F}_{\mathbb{F}} T: \mathrm{H}_{c}^{i}(\bar{X}, \mathcal{F})\right)^{(-1)^{i+1}} .
$$

It was used by Grothendieck to establish the rationality and the functional equation of the zeta function of $X$, both of which are parts of the Weil conjectures.

The Grothendieck trace formula may also be viewed as an equality between two elements of the first K-group of the power series ring $\mathbb{Z}_{\ell}[[T]]$. Since the ring $\mathbb{Z}_{\ell}[[T]]$ is a semilocal commutative ring, $\mathrm{K}_{1}\left(\mathbb{Z}_{\ell}[[T]]\right)$ may be identified with the group of units $\mathbb{Z}_{\ell}[[T]]^{\times}$via the map induced by the determinant. For each closed point $x$ of $X$, the $\mathbb{Z}_{\ell}[[T]]$-automorphism $1-\mathfrak{F}_{x} T$ on $\mathbb{Z}_{\ell}[[T]] \otimes_{\mathbb{Z}_{\ell}} \mathcal{F}_{x}$ defines a class in $\mathrm{K}_{1}\left(\mathbb{Z}_{\ell}[[T]]\right)$. The product of all these classes converges in the profinite topology induced on $\mathrm{K}_{1}\left(\mathbb{Z}_{\ell}[[T]]\right)$ by the isomorphism

$$
\mathrm{K}_{1}\left(\mathbb{Z}_{\ell}[[T]]\right) \cong \underset{n}{\lim } \mathrm{K}_{1}\left(\mathbb{Z}_{\ell}[[T]] /\left(\ell^{n}, T^{n}\right)\right)
$$

The image of the limit under the determinant map agrees with the inverse of the $L$-function of $\mathcal{F}$. On the other hand, the $\mathbb{Z}_{\ell}[[T]]$-automorphisms

$$
\mathbb{Z}_{\ell}[[T]] \otimes_{\mathbb{Z}_{\ell}} \mathrm{H}_{c}^{i}(\bar{X}, \mathcal{F}) \stackrel{1-\widetilde{\mathfrak{F}}_{\mathbb{F}} T}{\longrightarrow} \mathbb{Z}_{\ell}[[T]] \otimes_{\mathbb{Z}_{\ell}} \mathrm{H}_{c}^{i}(\bar{X}, \mathcal{F})
$$

also give rise to elements in the group $\mathrm{K}_{1}\left(\mathbb{Z}_{\ell}[[T]]\right)$. The Grothendieck trace formula may thus be translated into an equality between the alternating product of those elements and the class corresponding to the $L$-function.

In this article, we will show that in the above reformulation of the Grothendieck trace formula, one may replace $\mathbb{Z}_{\ell}$ by any adic $\mathbb{Z}_{\ell}$-algebra, i. e. a compact, semilocal

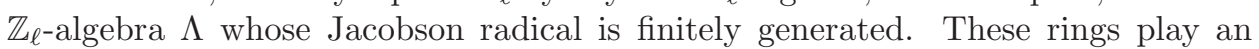
important role in noncommutative Iwasawa theory.

Date: November 2, 2018.

1991 Mathematics Subject Classification. 14G10 (11G25 14G15). 
A central step in this reformation is the development of a convenient framework, in which one can put the K-theoretic machinery to use. This was accomplished in Wit08, using the notion of Waldhausen categories. For any adic ring $\Lambda$, we introduced in loc. cit. a Waldhausen category of perfect complexes of adic sheaves of $\Lambda$ modules on $X$. Furthermore, we presented an explicit construction of a Waldhausen exact functor $\mathrm{R} \Gamma_{c}\left(\bar{X}, \mathcal{F}^{\bullet}\right)$ that computes the cohomology with proper support for any perfect complex $\mathcal{F}^{\bullet}$.

By suitably adapting the classical construction, we define the $L$-function of such a complex $\mathcal{F}^{\bullet}$ as an element $L\left(\mathcal{F}^{\bullet}, T\right)$ of $\mathrm{K}_{1}(\Lambda[[T]])$. The automorphism $1-\mathfrak{F}_{\mathbb{F}} T$ on $\Lambda[[T]] \otimes_{\Lambda} \mathrm{R} \Gamma_{c}\left(\bar{X}, \mathcal{F}^{\bullet}\right)$ gives rise to another class in $\mathrm{K}_{1}(\Lambda[[T]])$. Below, we shall prove the following theorem.

Theorem 1.1. Let $\mathcal{F}^{\bullet}$ be a perfect complex of adic sheaves of $\Lambda$-modules on $X$. Then

$$
L\left(\mathcal{F}^{\bullet}, T\right)=\left[\Lambda[[T]] \otimes_{\Lambda} \mathrm{R} \Gamma_{c}\left(\bar{X}, \mathcal{F}^{\bullet}\right) \stackrel{1-\mathfrak{F}_{\mathbb{F}} T}{\longrightarrow} \Lambda[[T]] \otimes_{\Lambda} \mathrm{R} \Gamma_{c}\left(\bar{X}, \mathcal{F}^{\bullet}\right)\right]^{-1}
$$

in $\mathrm{K}_{1}(\Lambda[[T]])$.

The rough line of argumentation in the proof is as follows. As in the proof of the classical Grothendieck trace formula, one may reduce everything to the case of $X$ being a smooth geometrically connected curve over the finite field $\mathbb{F}$. Moreover, one can replace $\Lambda$ by $\mathbb{Z}_{\ell}[\operatorname{Gal}(L / K)]$, where $L$ is a Galois extension of the function field $K$ of $X$. By the classical Grothendieck trace formula, we know that our theorem is true if we further enlarge $\mathbb{Z}_{\ell}[\operatorname{Gal}(L / K)]$ to the maximal order $M$ in a split semisimple algebra. The crucial step is then to show that the kernel of

$$
\mathrm{K}_{1}\left(\mathbb{Z}_{\ell}[\operatorname{Gal}(L / K)][[T]]\right) \rightarrow \mathrm{K}_{1}(M[[T]])
$$

vanishes in the limit as $L$ tends to the separable closure of $K$. This is achieved by using results of Oli88 and FK06.

Outline. Section 2 recalls briefly Waldhausen's construction of algebraic Ktheory. In Section 3 we introduce a special Waldhausen category that computes the K-theory of an adic ring. A similar construction is then used in Section 4 to define the categories of perfect complexes of adic sheaves. In Section 5 we study the first K-group of $\mathbb{Z}_{\ell}[G][[T]]$ and prove the abovementioned vanishing result. In Section 6 we define the $L$-function of a perfect complex of adic sheaves. Section 7 contains the proof of the Grothendieck trace formula for these $L$-functions.

Acknowledgements. The author would like to thank Annette Huber and Alexander Schmidt for their encouragement and for valuable discussions.

\section{Waldhausen Categories}

Waldhausen Wal85 introduced a construction of algebraic K-theory that is both more transparent and more flexible than Quillen's original approach. He associates $\mathrm{K}$-groups to any category of the following kind.

Definition 2.1. A Waldhausen category $\mathbf{W}$ is a category with a zero object $*$, together with two subcategories $\mathrm{co}(\mathbf{W})$ (cofibrations) and $\mathrm{w}(\mathbf{W})$ (weak equivalences) subject to the following set of axioms.

(1) Any isomorphism in $\mathbf{W}$ is a morphism in $\operatorname{co}(\mathbf{W})$ and $\mathrm{w}(\mathbf{W})$.

(2) For every object $A$ in $\mathbf{W}$, the unique map $* \rightarrow A$ is in $\operatorname{co}(\mathbf{W})$.

(3) If $A \rightarrow B$ is a map in $\operatorname{co}(\mathbf{W})$ and $A \rightarrow C$ is a map in $\mathbf{W}$, then the pushout $B \cup_{A} C$ exists and the canonical map $C \rightarrow B \cup_{A} C$ is in $\operatorname{co}(\mathbf{W})$. 
(4) If in the commutative diagram

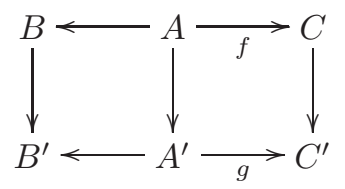

the morphisms $f$ and $g$ are cofibrations and the downwards pointing arrows are weak equivalences, then the natural map $B \cup_{A} C \rightarrow B^{\prime} \cup_{A^{\prime}} C^{\prime}$ is a weak equivalence.

We denote maps from $A$ to $B$ in $\operatorname{co}(\mathbf{W})$ by $A \longmapsto B$, those in w(W) by $A \stackrel{\sim}{\rightarrow} B$. If $C=B \cup_{A} *$ is a cokernel of the cofibration $A \longmapsto B$, then we denote the natural quotient map from $B$ to $C$ by $B \rightarrow C$. The sequence

$$
A \longmapsto B \rightarrow C
$$

is called exact sequence or cofibre sequence.

Definition 2.2. A functor between Waldhausen categories is called (Waldhausen) exact if it preserves cofibrations, weak equivalences, and pushouts along cofibrations.

If W is a Waldhausen category, then Waldhausen's $S$-construction yields a topological space $\mathbb{K}(\mathbf{W})$ and Waldhausen exact functors $F: \mathbf{W} \rightarrow \mathbf{W}^{\prime}$ yield continuous $\operatorname{maps} \mathbb{K}(F): \mathbb{K}(\mathbf{W}) \rightarrow \mathbb{K}\left(\mathbf{W}^{\prime}\right)$ Wal85.

Definition 2.3. The $n$-th $\mathrm{K}$-group of $\mathbf{W}$ is defined to be the $n$-th homotopy group of $\mathbb{K}(\mathbf{W})$ :

Example 2.4.

$$
\mathrm{K}_{n}(\mathbf{W})=\pi_{n}(\mathbb{K}(\mathbf{W}))
$$

(1) Any exact category $\mathbf{E}$ may be viewed as a Waldhausen category by taking the admissible monomorphisms as cofibrations and isomorphisms as weak equivalences. Then the Waldhausen K-groups of $\mathbf{E}$ agree with the Quillen K-groups of E [TT90, Theorem 1.11.2].

(2) $\operatorname{Let}_{\mathbf{K} \mathbf{o m}^{b}}(\mathbf{E})$ be the category of bounded complexes over the exact category $\mathbf{E}$ with degreewise admissible monomorphisms as cofibrations and quasi-isomorphisms (in the category of complexes of an ambient abelian category A) as weak equivalences. By the Gillet-Waldhausen theorem TT90, Theorem 1.11.7], the Waldhausen K-groups of $\mathbf{K o m}^{b}(\mathbf{E})$ also agree with the K-groups of $\mathbf{E}$.

(3) In fact, Thomason showed that if $\mathbf{W}$ is any sufficiently nice Waldhausen category of complexes and $F: \mathbf{W} \rightarrow \mathbf{K o m}^{b}(\mathbf{E})$ a Waldhausen exact functor that induces an equivalence of the derived categories of $\mathbf{W}$ and $\mathbf{K o m}^{b}(\mathbf{E})$, then $F$ induces an isomorphism of the corresponding K-groups [TT90, Theorem 1.9.8].

Remark 2.5. In the view of Example 2.4. (3) one might wonder wether it is possible to define a reasonable K-theory for triangulated categories. However, Sch02 shows that such a construction fails to exist.

The zeroth K-group of a Waldhausen category can be described fairly explicitly as follows.

Proposition 2.6. Let $\mathbf{W}$ be a Waldhausen category. The group $\mathrm{K}_{0}(\mathbf{W})$ is the abelian group generated by the objects of $\mathbf{W}$ modulo the relations

(1) $[A]=[B]$ if there exists a weak equivalence $A \stackrel{\sim}{\rightarrow} B$, 
(2) $[B]=[A][C]$ if there exists a cofibre sequence $A \longmapsto B \rightarrow C$.

Proof. See [TT90, §1.5.6].

There also exists a description of $K_{1}(\mathbf{W})$ for general $\mathbf{W}$ as the kernel of a certain group homomorphism MT07. We shall come back to this description later in a more specific situation.

\section{The K-Theory of Adic Rings}

All rings will be associative with unity, but not necessarily commutative. For any ring $R$, we let

$$
\operatorname{Jac}(R)=\{x \in R \mid 1-r x \text { is invertible for any } r \in R\}
$$

denote the Jacobson radical of $R$, i. e. the intersection of all maximal left ideals. It is the largest two-sided ideal $I$ of $R$ such that $1+I \subset R^{\times}$[Lam91, Chapter 2, §4]. The ring $R$ is called semilocal if $R / \operatorname{Jac}(R)$ is artinian.

Definition 3.1. A ring $\Lambda$ is called an adic ring if it satisfies any of the following equivalent conditions:

(1) $\Lambda$ is compact, semilocal and the Jacobson radical is finitely generated.

(2) For each integer $n \geq 1$, the ideal $\operatorname{Jac}(\Lambda)^{n}$ is of finite index in $\Lambda$ and

$$
\Lambda=\lim _{n} \Lambda / \operatorname{Jac}(\Lambda)^{n} \text {. }
$$

(3) There exists a twosided ideal $I$ such that for each integer $n \geq 1$, the ideal $I^{n}$ is of finite index in $\Lambda$ and

$$
\Lambda=\lim _{n} \Lambda / I^{n} \text {. }
$$

Example 3.2. The following rings are adic rings:

(1) any finite ring,

(2) $\mathbb{Z}_{\ell}$,

(3) the group $\operatorname{ring} \Lambda[G]$ for any finite group $G$ and any adic $\operatorname{ring} \Lambda$,

(4) the power series ring $\Lambda[[T]]$ for any adic $\operatorname{ring} \Lambda$ and an indeterminate $T$ that commutes with all elements of $\Lambda$,

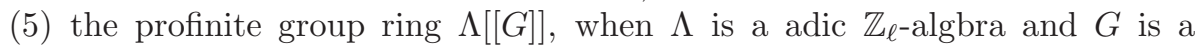
profinite group whose $\ell$-Sylow subgroup has finite index in $G$.

Note that adic rings are not noetherian in general, the power series over $\mathbb{Z}_{\ell}$ in two noncommuting indeterminates being a counterexample.

We will now examine the K-theory of $\Lambda$.

Definition 3.3. Let $R$ be any ring. A complex $M^{\bullet}$ of left $R$-modules is called strictly perfect if it is strictly bounded and for every $n$, the module $M^{n}$ is finitely generated and projective. We let $\mathbf{S P}(R)$ denote the Waldhausen category of strictly perfect complexes, with quasi-isomorphisms as weak equivalences and injective complex morphisms as cofibrations.

Definition 3.4. Let $R$ and $S$ be two rings. We denote by $R^{o p}-\mathbf{S P}(S)$ the Waldhausen category of complexes of $S$-R-bimodules (with $S$ acting from the left, $R$ acting from the right) which are strictly perfect as complexes of $S$-modules. The weak equivalences are given by quasi-isomorphisms, the cofibrations are the injective complex morphisms. 
By Example 2.4 we know that the Waldhausen K-theory of $\mathbf{S P}(R)$ coincides with the Quillen K-theory of $R$ :

$$
\mathrm{K}_{n}(\mathbf{S P}(R))=\mathrm{K}_{n}(R) .
$$

For complexes $M^{\bullet}$ and $N^{\bullet}$ of right and left $R$-modules, respectively, we let

$$
\left(M \otimes_{R} N\right)^{\bullet}
$$

denote the total complex of the bicomplex $M^{\bullet} \otimes_{R} N^{\bullet}$. Any complex $M^{\bullet}$ in $R^{o p}-\mathbf{S P}(S)$ clearly gives rise to a Waldhausen exact functor

$$
\left(M \otimes_{R}(-)\right)^{\bullet}: \mathbf{S P}(R) \rightarrow \mathbf{S P}(S) .
$$

and hence, to homomorphisms $\mathrm{K}_{n}(R) \rightarrow \mathrm{K}_{n}(S)$.

Let now $\Lambda$ be an adic ring. The first algebraic K-group of $\Lambda$ has the following useful property.

Proposition 3.5 ([FK06], Prop. 1.5.3). Let $\Lambda$ be an adic ring. Then

$$
\mathrm{K}_{1}(\Lambda)=\lim _{I \in \mathfrak{I}_{\Lambda}} \mathrm{K}_{1}(\Lambda / I)
$$

In particular, $\mathrm{K}_{1}(\Lambda)$ is a profinite group.

It will be convenient to introduce another Waldhausen category that computes the K-theory of $\Lambda$.

Definition 3.6. Let $R$ be any ring. A complex $M^{\bullet}$ of left $R$-modules is called $D G$ flat if every module $M^{n}$ is flat and for every acyclic complex $N^{\bullet}$ of right $R$-modules, the complex $\left(N \otimes_{R} M\right)^{\bullet}$ is acyclic.

We shall denote the lattice of open ideals of an adic $\operatorname{ring} \Lambda$ by $\mathfrak{I}_{\Lambda}$.

Definition 3.7. Let $\Lambda$ be an adic ring. We denote by $\mathbf{P D G}{ }^{\text {cont }}(\Lambda)$ the following Waldhausen category. The objects of $\mathbf{P D G}{ }^{\text {cont }}(\Lambda)$ are inverse system $\left(P_{I}^{\bullet}\right)_{I \in \mathfrak{I}_{\Lambda}}$ satifying the following conditions:

(1) for each $I \in \mathfrak{I}_{\Lambda}, P_{I}^{\bullet}$ is a $D G$-flat complex of left $\Lambda / I$-modules and perfect, i. e. quasi-isomorphic to a complex in $\mathbf{S P}(\Lambda)$,

(2) for each $I \subset J \in \mathfrak{I}_{\Lambda}$, the transition morphism of the system

$$
\varphi_{I J}: P_{I}^{\bullet} \rightarrow P_{J}^{\bullet}
$$

induces an isomorphism

$$
\Lambda / J \otimes_{\Lambda / I} P_{I}^{\bullet} \cong P_{J}^{\bullet}
$$

A morphism of inverse systems $\left(f_{I}: P_{I}^{\bullet} \rightarrow Q_{I}^{\bullet}\right)_{I \in \mathfrak{I}_{\Lambda}}$ in $\mathbf{P D G} \mathbf{D G}^{\text {cont }}(\Lambda)$ is a weak equivalence if every $f_{I}$ is a quasi-isomorphism. It is a cofibration if every $f_{I}$ is injective.

The following proposition is an easy consequence of Waldhausen's approximation theorem.

Proposition 3.8. The Waldhausen exact functor

$$
\operatorname{SP}(\Lambda) \rightarrow \mathbf{P D G}^{\text {cont }}(\Lambda), \quad P^{\bullet} \rightarrow\left(\Lambda / I \otimes_{\Lambda} P^{\bullet}\right)_{I \in \mathfrak{I}_{\Lambda}}
$$

identifies $\mathbf{S P}(\Lambda)$ with a full Waldhausen subcategory of $\mathbf{P D G}{ }^{\text {cont }}(\Lambda)$. Moreover, it induces isomorphisms

$$
\mathrm{K}_{n}(\mathbf{S P}(\Lambda)) \cong \mathrm{K}_{n}\left(\mathbf{P D G}^{\text {cont }}(\Lambda)\right)
$$

Proof. See Wit08, Proposition 5.2.5].

We will now extend the definition of the tensor product to $\mathbf{P D G}{ }^{\text {cont }}(\Lambda)$. 
Definition 3.9. For $\left(P_{I}^{\bullet}\right)_{I \in \mathfrak{I}_{\Lambda}} \in \mathbf{P D G}^{\text {cont }}(\Lambda)$ and $M^{\bullet} \in \Lambda^{o p} \mathbf{S P}_{-}\left(\Lambda^{\prime}\right)$ we set

$$
\Psi_{M}\left(\left(P_{I}^{\bullet}\right)_{I \in \mathfrak{I}_{\Lambda}}\right)=\left(\lim _{J \in \mathfrak{I}_{\Lambda}} \Lambda^{\prime} / I \otimes_{\Lambda^{\prime}}\left(M \otimes_{\Lambda} P_{J}\right)^{\bullet}\right)_{I \in \mathfrak{I}_{\Lambda^{\prime}}}
$$

and obtain a Waldhausen exact functor

$$
\Psi_{M}: \mathbf{P D G}^{\text {cont }}(\Lambda) \rightarrow \mathbf{P D G}^{\text {cont }}\left(\Lambda^{\prime}\right) .
$$

Proposition 3.10. Let $M^{\bullet}$ be a complex in $\Lambda^{o p}{ }_{-} \mathbf{S P}\left(\Lambda^{\prime}\right)$. Then the following diagram commutes.

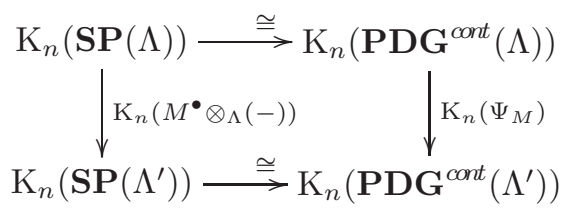

Proof. Let $P^{\bullet}$ be a strictly perfect complex in $\mathbf{S P}(\Lambda)$. There exists a canonical isomorphism

$$
\left(\Lambda^{\prime} / I \otimes_{\Lambda^{\prime}}\left(M \otimes_{\Lambda} P\right)^{\bullet}\right)_{I \in \mathfrak{I}_{\Lambda^{\prime}}} \cong\left(\lim _{J \in \mathfrak{I}_{\Lambda}} \Lambda^{\prime} / I \otimes_{\Lambda^{\prime}}\left(M \otimes_{\Lambda} \Lambda / J \otimes_{\Lambda} P\right)^{\bullet}\right)_{I \in \mathfrak{I}_{\Lambda^{\prime}}}
$$

From MT07] we deduce the following properties of the group $K_{1}(\Lambda)$.

Proposition 3.11. The group $\mathrm{K}_{1}(\Lambda)$ is generated by the weak autoequivalences $\left(f_{I}: P_{I}^{\bullet} \stackrel{\sim}{\longrightarrow} P_{I}^{\bullet}\right)_{I \in \mathfrak{I}_{\Lambda}}$ in $\mathbf{P D G}{ }^{\text {cont }}(\Lambda)$. Moreover, we have the following relations:

(1) $\left[\left(f_{I}: P_{I}^{\bullet} \stackrel{\sim}{\rightarrow} P_{I}^{\bullet}\right)_{I \in \mathfrak{I}_{\Lambda}}\right]=\left[\left(g_{I}: P_{I}^{\bullet} \stackrel{\sim}{\longrightarrow} P_{I}^{\bullet}\right)_{I \in \mathfrak{I}_{\Lambda}}\right]\left[\left(h_{I}: P_{I}^{\bullet} \stackrel{\sim}{\rightarrow} P_{I}^{\bullet}\right)_{I \in \mathfrak{I}_{\Lambda}}\right]$ if for each $I \in \mathfrak{I}_{\Lambda}$, one has $f_{I}=g_{I} \circ h_{I}$,

(2) $\left[\left(f_{I}: P_{I}^{\bullet} \stackrel{\sim}{\rightarrow} P_{I}^{\bullet}\right)_{I \in \mathfrak{I}_{\Lambda}}\right]=\left[\left(g_{I}: Q_{I}^{\bullet} \stackrel{\sim}{\longrightarrow} Q_{I}^{\bullet}\right)_{I \in \mathfrak{I}_{\Lambda}}\right]$ if for each $I \in \mathfrak{I}_{\Lambda}$, there exists a quasi-isomorphism $a_{I}: P_{I}^{\bullet} \stackrel{\sim}{\longrightarrow} Q_{I}^{\bullet}$ such that the square

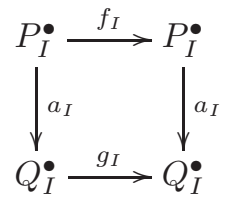

commutes up to homotopy,

(3) $\left[\left(g_{I}: P_{I}^{\prime \bullet} \stackrel{\sim}{\longrightarrow} P_{I}^{\prime \bullet}\right)_{I \in \mathfrak{I}_{\Lambda}}\right]=\left[\left(f_{I}: P_{I}^{\bullet} \stackrel{\sim}{\rightarrow} P_{I}^{\bullet}\right)_{I \in \mathfrak{I}_{\Lambda}}\right]\left[\left(h_{I}: P_{I}^{\prime \prime \bullet} \stackrel{\sim}{\rightarrow} P_{I}^{\prime \prime \bullet}\right)_{I \in \mathfrak{I}_{\Lambda}}\right]$ if for each $I \in \mathfrak{I}_{\Lambda}$, there exists an exact sequence $P \longmapsto P^{\prime} \rightarrow P^{\prime \prime}$ such that the diagram

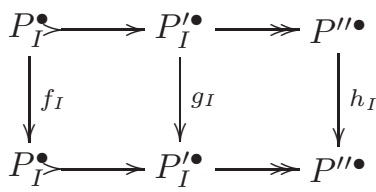

commutes in the strict sense.

Proof. The description of $\mathrm{K}_{1}\left(\mathbf{P D G}{ }^{\text {cont }}(\Lambda)\right)$ as the kernel of

$$
\mathcal{D}_{1} \mathbf{P D G}{ }^{\text {cont }}(\Lambda) \stackrel{\partial}{\rightarrow} \mathcal{D}_{0} \mathbf{P D G}{ }^{\text {cont }}(\Lambda)
$$

given in MT07 shows that the weak autoequivalences are indeed elements of $\mathrm{K}_{1}\left(\mathbf{P D G}^{\text {cont }}(\Lambda)\right)$. Together with Proposition 3.5 this description also implies that relations (1) and (3) are satisfied. For relation (2), one can use Wit08, Lemma 3.1.6]. Finally, the classical description of $K_{1}(\Lambda)$ implies that $K_{1}\left(\mathbf{P D G}^{\text {cont }}(\Lambda)\right)$ is already generated by isomorphisms of finitely generated, projective modules viewed as strictly perfect complexes concentrated in degree 0 . 
Remark 3.12. Despite the relatively explicit description of $K_{1}(\mathbf{W})$ for a Waldhausen category $\mathbf{W}$ in [MT07] it is not an easy task to deduce from it a presentation of $\mathrm{K}_{1}(\mathbf{W})$ as an abelian group. We refer to MT08 for a partial result in this direction.

In particular, one should not expect that the relations (1)-(3) describe the group $\mathrm{K}_{1}\left(\mathbf{P D G}^{\text {cont }}(\Lambda)\right)$ completely. However, they will suffice for the purpose of this paper.

\section{Perfect Complexes of Adic Sheaves}

We let $\mathbb{F}$ denote a finite field of characteristic $p$, with $q=p^{\nu}$ elements. Furthermore, we fix an algebraic closure $\overline{\mathbb{F}}$ of $\mathbb{F}$.

For any scheme $X$ in the category $\mathbf{S c h}_{\mathbb{F}}^{\text {sep }}$ of separated $\mathbb{F}$-schemes of finite type and any adic ring $\Lambda$ we introduced in Wit08, a Waldhausen category $\mathbf{P D G}^{\text {cont }}(X, \Lambda)$ of perfect complexes of adic sheaves on $X$. Below, we will recall the definition.

Definition 4.1. Let $R$ be a finite ring and $X$ be a scheme in $\mathbf{S c h}_{\mathbb{F}}^{\text {sep }}$. A complex $\mathcal{F}^{\bullet}$ of étale sheaves of left $R$-modules on $X$ is called strictly perfect if it is strictly bounded and each $\mathcal{F}^{n}$ is constructible and flat. A complex is called perfect if it is quasi-isomorphic to a strictly perfect complex. It is $D G$-flat if for each geometric point of $X$, the complex of stalks is $D G$-flat.

Definition 4.2. We will denote by $\mathbf{P D G}(X, R)$ the category of DG-flat perfect complexes of $R$-modules on $X$. It is a Waldhausen category with quasiisomorphisms as weak equivalences and injective complex morphisms as cofibrations.

Definition 4.3. Let $X$ be a scheme in $\mathbf{S c h}_{\mathbb{F}}$ and let $\Lambda$ be an adic ring. The category of perfect complexes of adic sheaves $\mathbf{P D G}{ }^{\text {cont }}(X, \Lambda)$ is the following Waldhausen category. The objects of $\mathbf{P D G}{ }^{\text {cont }}(X, \Lambda)$ are inverse system $\left(\mathcal{F}_{I}^{\bullet}\right)_{I \in \mathfrak{I}_{\Lambda}}$ such that:

(1) for each $I \in \mathfrak{I}_{\Lambda}, \mathcal{F}_{I}^{\bullet}$ is in $\mathbf{P D G}(X, \Lambda / I)$,

(2) for each $I \subset J \in \mathfrak{I}_{\Lambda}$, the transition morphism

$$
\varphi_{I J}: \mathcal{F}_{I}^{\bullet} \rightarrow \mathcal{F}_{J}^{\bullet}
$$

of the system induces an isomorphism

$$
\Lambda / J \otimes_{\Lambda / I} \mathcal{F}_{I}^{\bullet} \stackrel{\sim}{\longrightarrow} \mathcal{F}_{J}^{\bullet}
$$

Weak equivalences and cofibrations are those morphisms of inverse systems that are weak equivalences or cofibrations for each $I \in \mathfrak{I}_{\Lambda}$, respectively.

Remark 4.4. If $\Lambda$ is a finite ring, the zero ideal is open and hence, an element in $\Im_{\Lambda}$. In particular, the following Waldhausen exact functors are mutually inverse equivalences for finite rings $\Lambda$ :

$$
\begin{array}{ll}
\operatorname{PDG}^{\text {cont }}(X, \Lambda) \rightarrow \operatorname{PDG}(X, \Lambda), & \left(\mathcal{F}_{I}^{\bullet}\right)_{I \in \mathfrak{I}_{\Lambda}} \mapsto \mathcal{F}_{(0)}^{\bullet}, \\
\operatorname{PDG}(X, \Lambda) \rightarrow \mathbf{P D G}^{\text {cont }}(X, \Lambda), & \mathcal{F}^{\bullet} \mapsto\left(\Lambda / I \otimes_{\Lambda} \mathcal{F}^{\bullet}\right)_{I \in \mathfrak{I}_{\Lambda}} .
\end{array}
$$

We use these functors to identify the two categories.

If $\Lambda=\mathbb{Z}_{\ell}$, then the subcategory of complexes concentrated in degree 0 of $\mathbf{P D G}^{\text {cont }}\left(X, \mathbb{Z}_{\ell}\right)$ corresponds precisely to the exact category of flat constructible $\ell$-adic sheaves on $X$ in the sense of [Gro77, Exposé VI, Definition 1.1.1]. In this sense, we recover the classical theory.

If $f: Y \rightarrow X$ is a morphism of schemes, we define a Waldhausen exact functor

$$
f^{*}: \mathbf{P D G}^{\text {cont }}(X, \Lambda) \rightarrow \mathbf{P D G}^{\text {cont }}(Y, \Lambda), \quad\left(\mathcal{F}_{I}^{\bullet}\right)_{I \in \mathfrak{I}_{\Lambda}} \mapsto\left(f^{*} \mathcal{F}_{I}^{\bullet}\right)_{I \in \mathfrak{I}_{\Lambda}} .
$$

We will also need a Waldhausen exact functor that computes higher direct images with proper support. For the purposes of this article it suffices to use the following construction. 
Definition 4.5. Let $f: X \rightarrow Y$ be a morphism in $\mathbf{S c h}_{\mathbb{F}}^{s e p}$. Then there exists a factorisation $f=p \circ j$ with $j: X \leftrightarrow X^{\prime}$ an open immersion and $p: X^{\prime} \rightarrow Y$ a proper morphism. Let $\mathrm{G}_{X^{\prime}}^{\bullet} \mathcal{G}$ denote the Godement resolution of a complex $\mathcal{G}^{\bullet}$ of abelian étale sheaves on $X^{\prime}$. Define

$$
\begin{aligned}
& \mathrm{R} f !_{!}: \mathbf{P D G}^{\text {cont }}(X, \Lambda) \rightarrow \mathbf{P D G}^{\text {cont }}(Y, \Lambda) \\
&\left(\mathcal{F}_{I}^{\bullet}\right)_{I \in \mathfrak{I}_{\Lambda}} \mapsto\left(f_{*} \mathrm{G}_{X^{\prime}}^{\bullet} j_{!} \mathcal{F}_{I}\right)_{I \in \mathfrak{I}_{\Lambda}}
\end{aligned}
$$

Obviously, this definition depends on the particular choice of the compactification $f=p \circ j$. However, all possible choices will induce the same homomorphisms

$$
\mathrm{K}_{n}\left(\mathrm{R}_{\mathrm{l}}\right): \mathrm{K}_{n}\left(\mathbf{P D G}^{\text {cont }}(X, \Lambda)\right) \rightarrow \mathrm{K}_{n}\left(\mathbf{P D G}^{\text {cont }}(Y, \Lambda)\right)
$$

and this is all we need.

Remark 4.6. In Wit08, Section 4.5] we present a way to make the construction of $\mathrm{R} f$ ! independent of the choice of a particular compactification.

Proposition 4.7. Let $f: X \rightarrow Y$ be a morphism in $\mathbf{S} \mathbf{c h}_{\mathbb{F}}^{\text {sep }}$.

(1) $\mathrm{K}_{n}\left(\mathrm{R} f_{!}\right)$is independent of the choice of a compactification $f=p \circ j$.

(2) Let $\mathbb{F}^{\prime}$ be a subfield of $\mathbb{F}$ and consider $f$ as a morphism in $\mathbf{S c h}_{\mathbb{F}^{\prime}}^{\text {sep }}$. Then $\mathrm{K}_{n}\left(\mathrm{R} f_{!}\right)$remains the same.

(3) If $g: Y \rightarrow Z$ is another morphism in $\mathbf{S c h}_{\mathbb{F}}^{\text {sep }}$, then

$$
\mathrm{K}_{n}(\mathrm{R}(g \circ f) !)=\mathrm{K}_{n}\left(\mathrm{R} g_{!}\right) \circ \mathrm{K}_{n}\left(\mathrm{R} f_{!}\right)
$$

(4) For any cartesian square

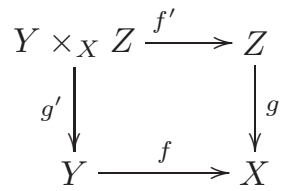

in $\mathbf{S c h}_{\mathbb{F}}^{\text {sep }}$ we have

$$
\mathrm{K}_{n}\left(f^{*} \mathrm{R} g_{!}\right)=\mathrm{K}_{n}\left(\mathrm{R} g_{!}^{\prime} f^{\prime *}\right)
$$

Proof. All of this follows easily from AGV72, Exposé XXVII]. See also Wit08, Section 4.5.].

Definition 4.8. Let $X$ be a scheme in $\mathbf{S c h}_{\mathbb{F}}^{\text {sep }}$ and write $h: X \rightarrow \operatorname{Spec} \mathbb{F}$ for the structure map, $s:$ Spec $\overline{\mathbb{F}} \rightarrow$ Spec $\mathbb{F}$ for the map induced by the embedding into the algebraic closure. We define the Waldhausen exact functor

$$
\mathrm{R}_{\mathbb{F}} \Gamma_{c}(\bar{X},-): \mathbf{P D G}^{\text {cont }}(X, \Lambda) \rightarrow \mathbf{P D G}^{\text {cont }}(\Lambda)
$$

to be the composition of

$$
\mathrm{R} h_{!}: \mathbf{P D G}^{\text {cont }}(X, \Lambda) \rightarrow \mathbf{P D G}^{\text {cont }}(\operatorname{Spec} \mathbb{F}, \Lambda)
$$

with the section functor

$$
\mathbf{P D G}^{\text {cont }}(\operatorname{Spec} \mathbb{F}, \Lambda) \rightarrow \mathbf{P D G}^{\text {cont }}(\Lambda), \quad\left(\mathcal{F}_{I}^{\bullet}\right)_{I \in \mathfrak{I}_{\Lambda}} \rightarrow\left(\Gamma\left(\operatorname{Spec} \overline{\mathbb{F}}, s^{*} \mathcal{F}_{I}^{\bullet}\right)\right)_{I \in \mathfrak{I}_{\Lambda}} .
$$

Remark 4.9. If $\mathbb{F}^{\prime}$ is a subfield of $\mathbb{F}$, then $\mathrm{R}_{\mathbb{F}} \Gamma_{c}(\bar{X},-)$ and $\mathrm{R}_{\mathbb{F}^{\prime}} \Gamma_{c}(\bar{X},-)$ are in fact quasi-isomorphic and hence, they induce the same homomorphism of K-groups. Nevertheless, it will be convenient to distinguish between the two functors. We will omit the index if the base field is clear from the context.

The definition of $\Psi_{M}$ extends to $\mathbf{P D G}{ }^{\text {cont }}(X, \Lambda)$. 
Definition 4.10. For $\left(\mathcal{F}_{I}^{\bullet}\right)_{I \in \mathfrak{I}_{\Lambda}} \in \mathbf{P D G}^{\text {cont }}(X, \Lambda)$ and $M^{\bullet} \in \Lambda^{o p}-\mathbf{S P}\left(\Lambda^{\prime}\right)$ we set

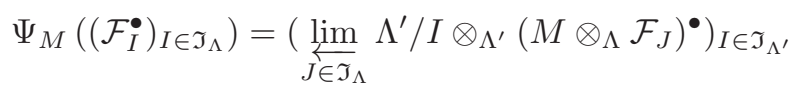

and obtain a Waldhausen exact functor

$$
\Psi_{M}: \mathbf{P D G}^{\text {cont }}(X, \Lambda) \rightarrow \mathbf{P D G}^{\text {cont }}\left(X, \Lambda^{\prime}\right) .
$$

Proposition 4.11. Let $M^{\bullet}$ be a complex in $\Lambda^{o p}{ }_{-} \mathbf{S P}\left(\Lambda^{\prime}\right)$. Then the following diagram commutes.

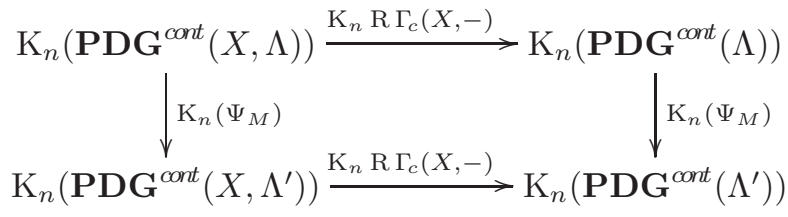

Proof. See [Wit08, Proposition 5.5.7].

Finally, we need the following result. Let $X$ be a connected scheme and $f: Y \rightarrow$ $X$ a finite Galois covering of $X$ with Galois group $G$, i. e. $f$ is finite étale and the degree of $f$ is equal to the order of $G=\operatorname{Aut}_{X}(Y)$. We set

$$
\mathbb{Z}_{\ell}[G]_{X}^{\sharp}=f_{!} f^{*} \mathbb{Z}_{\ell} \text {. }
$$

Then $\mathbb{Z}_{\ell}[G]_{X}^{\sharp}$ is a locally constant constructible flat sheaf of $\mathbb{Z}_{\ell}[G]$-modules. In fact, it corresponds to the continous Galois module $\mathbb{Z}_{\ell}[G]$ on which the fundamental group of $X$ acts contragrediently.

Lemma 4.12. Let $X$ be a connected scheme in $\mathbf{S c h}_{\mathbb{F}}^{\text {sep }}$. Let $R$ be a finite $\mathbb{Z}_{\ell}$-algebra and let $\mathcal{F}^{\bullet}$ be a bounded complex of flat, locally constant, and constructible sheaves in $\mathbf{P D G}(X, R)$. Then there exists a finite Galois covering $Y$ of $X$ with Galois group $G$ and a complex $M^{\bullet}$ in $\mathbb{Z}_{\ell}[G]^{o p}{ }_{-} \mathbf{S P}(R)$ such that

$$
\mathcal{F}^{\bullet} \cong \Psi_{M}\left(\mathbb{Z}_{\ell}[G]_{X}^{\sharp}\right) \text {. }
$$

Proof. Choose a large enough Galois covering $f: Y \rightarrow X$ such that $f^{*} \mathcal{F}^{\bullet}$ is a complex of constant sheaves and set $M^{\bullet}=\Gamma\left(Y, f^{*} \mathcal{F}^{\bullet}\right)$. This is in a natural way a complex in $\mathbb{Z}_{\ell}[G]^{o p}$-SP $(R)$ and

$$
M^{\bullet} \otimes_{\mathbb{Z}_{\ell}[G]} \mathbb{Z}_{\ell}[G]_{X}^{\sharp} \cong \mathcal{F}^{\bullet}
$$

See [Wit08, Section 5.6] for further details.

\section{ON $\mathrm{K}_{1}\left(\mathbb{Z}_{\ell}[G][[T]]\right)$}

Let $G$ be a finite group and let $T$ denote an indeterminate that commutes with every element of $\mathbb{Z}_{\ell}[G]$. We need some results on the structure of $\mathrm{K}_{1}\left(\mathbb{Z}_{\ell}[G][[T]]\right)$. Recall that there exists a finite extension $F$ of $\mathbb{Q}_{\ell}$ such that $F[G]$ is split semisimiple:

$$
F[G] \cong \prod_{k=1}^{r} \operatorname{End}_{F}\left(F^{s_{k}}\right)
$$

for some integers $r, s_{1}, \ldots, s_{r}$. Write $\mathcal{O}_{F}$ for the valuation $\operatorname{ring}$ of $F$ and let $M$ be a

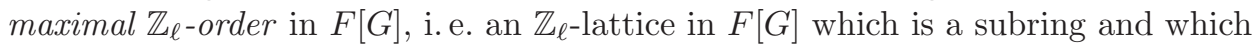
is maximal with respect to this property. Then

$$
M \cong \prod_{k=1}^{r} \operatorname{End}_{\mathcal{O}_{F}}\left(\mathcal{O}_{F}^{s_{k}}\right)
$$


according to Oli88, Theorem 1.9]. In particular, the determinant map induces an isomorphism

$$
\mathrm{K}_{1}(M) \cong \bigoplus_{k=1}^{r} \mathcal{O}_{F}^{\times} .
$$

Theorem 2.5 of loc. cit. then implies

$$
\operatorname{SK}_{1}\left(\mathbb{Z}_{\ell}[G]\right)=\operatorname{ker} \mathrm{K}_{1}\left(\mathbb{Z}_{\ell}[G]\right) \rightarrow \mathrm{K}_{1}\left(\mathbb{Q}_{\ell}[G]\right)=\operatorname{ker} \mathrm{K}_{1}\left(\mathbb{Z}_{\ell}[G]\right) \rightarrow \mathrm{K}_{1}(M) .
$$

Analogously, we define a subgroup in $\mathrm{K}_{1}\left(\mathbb{Z}_{\ell}[G][[T]]\right)$.

Definition 5.1. Let $G$ be a finite group and choose a finite extension $F$ of $\mathbb{Q}_{\ell}$ such that $F[G]$ is split semisimple. We set

$$
\mathrm{SK}_{1}\left(\mathbb{Z}_{\ell}[G][[T]]\right)=\operatorname{ker} \mathrm{K}_{1}\left(\mathbb{Z}_{\ell}[G][[T]]\right) \rightarrow \mathrm{K}_{1}(M[[T]])
$$

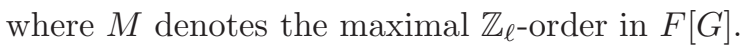

Lemma 5.2. For any finite group $G$,

$$
\mathrm{SK}_{1}\left(\mathbb{Z}_{\ell}[G][[T]]\right) \cong \underset{n}{\lim } \mathrm{SK}_{1}\left(\mathbb{Z}_{\ell}\left[G \times \mathbb{Z} /\left(\ell^{n}\right)\right]\right) .
$$

Proof. Let $F$ and $F^{\prime}$ be splitting fields for $\mathbb{Z}_{\ell}[G]$ and $\mathbb{Z}_{\ell}\left[G \times \mathbb{Z} /\left(\ell^{n}\right)\right]$, respectively and denote the corresponding maximal orders by $M$ and $M^{\prime}$. The commutativity of the diagram

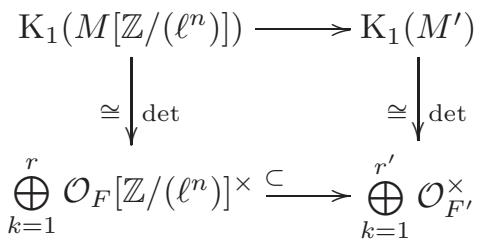

implies that

$$
\mathrm{SK}_{1}\left(\mathbb{Z}_{\ell}\left[G \times \mathbb{Z} /\left(\ell^{n}\right)\right]\right)=\operatorname{ker} \mathrm{K}_{1}\left(\mathbb{Z}_{\ell}\left[G \times \mathbb{Z} /\left(\ell^{n}\right)\right]\right) \rightarrow \mathrm{K}_{1}\left(M\left[\mathbb{Z} /\left(\ell^{n}\right)\right]\right) .
$$

By [NSW00, Theorem 5.3.5] the choice of a topological generator $\gamma \in \mathbb{Z}_{\ell}$ induces an isomorphism

$$
\mathbb{Z}_{\ell}[[T]] \cong \lim _{n} \mathbb{Z}_{\ell}\left[\mathbb{Z} /\left(\ell^{n}\right)\right], \quad T \mapsto \gamma-1
$$

In particular, we have compatible isomorphisms

$$
\mathrm{K}_{1}\left(\mathbb{Z}_{\ell}[G][[T]]\right) \cong \longleftarrow_{n}^{\lim } \mathrm{K}_{1}\left(\mathbb{Z}_{\ell}\left[G \times \mathbb{Z} /\left(\ell^{n}\right)\right]\right), \quad \mathrm{K}_{1}(M[[T]]) \cong{\underset{n}{n}}_{\lim } \mathrm{K}_{1}\left(M\left[\mathbb{Z} /\left(\ell^{n}\right)\right]\right) .
$$

by Proposition 3.5. Hence, we obtain an isomorphism

$$
\mathrm{SK}_{1}\left(\mathbb{Z}_{\ell}[G][[T]]\right) \cong \underset{n}{\lim } \mathrm{SK}_{1}\left(\mathbb{Z}_{\ell}\left[G \times \mathbb{Z} /\left(\ell^{n}\right)\right]\right),
$$

as claimed.

Proposition 5.3. For any finite group $G$,

$$
\mathrm{SK}_{1}\left(\mathbb{Z}_{\ell}[G][[T]]\right) \cong \mathrm{SK}_{1}\left(\mathbb{Z}_{\ell}[G]\right) .
$$

Proof. By Lemma 5.2 it suffices to prove that the projection $\mathbb{Z}_{\ell}\left[G \times \mathbb{Z} /\left(\ell^{n}\right)\right] \rightarrow \mathbb{Z}_{\ell}[G]$ induces an isomorphism

$$
\mathrm{SK}_{1}\left(\mathbb{Z}_{\ell}\left[G \times \mathbb{Z} /\left(\ell^{n}\right)\right]\right) \cong \mathrm{SK}_{1}\left(\mathbb{Z}_{\ell}[G]\right) .
$$

Let $g_{1}, \ldots, g_{k}$ be a system of representatives for the $\mathbb{Q}_{\ell}$-conjugacy classes of elements of order prime to $\ell$ in $G$. (Two elements $g, h$ of order $r$ prime to $\ell$ are called 


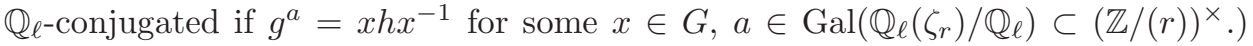
Let $r_{i}$ denote the order of $g_{i}$ and set

$$
\begin{aligned}
& N_{i}(G)=\left\{x \in G \quad \mid x g_{i} x^{-1}=g_{i}^{a} \text { for some } a \in \operatorname{Gal}\left(\mathbb{Q}_{\ell}\left(\zeta_{r_{i}}\right) / \mathbb{Q}_{\ell}\right)\right\}, \\
& Z_{i}(G)=\left\{x \in G \quad \mid x g_{i} x^{-1}=g_{i}\right\} .
\end{aligned}
$$

Furthermore, let

$$
\mathrm{H}_{2}^{a b}\left(Z_{i}(G), \mathbb{Z}\right)=\mathrm{im} \bigoplus_{\substack{H \subset Z_{i}(G) \\ \text { abelian }}} \mathrm{H}_{2}(H, \mathbb{Z}) \rightarrow \mathrm{H}_{2}\left(Z_{i}(G), \mathbb{Z}\right)
$$

denote the subgroup of the second homology group generated by elements induced up from abelian subgroups of $Z_{i}(G)$. According to [Oli88, Theorem 12.5] there exists an isomorphism

$$
\mathrm{SK}_{1}\left(\mathbb{Z}_{\ell}[G]\right) \cong \bigoplus_{i=0}^{k} \mathrm{H}_{0}\left(N_{i}(G) / Z_{i}(G), \mathrm{H}_{2}\left(Z_{i}(G), \mathbb{Z}\right) / \mathrm{H}_{2}^{a b}\left(Z_{i}(G), \mathbb{Z}\right)\right)_{(\ell)} .
$$

Now, $\left(g_{1}, 0\right), \ldots,\left(g_{k}, 0\right)$ is a system of representatives for the $\mathbb{Q}_{\ell}$-conjugacy classes of elements of order prime to $\ell$ in $G \times \mathbb{Z} /\left(\ell^{n}\right)$ and

$$
N_{i}\left(G \times \mathbb{Z} /\left(\ell^{n}\right)\right)=N_{i}(G) \times \mathbb{Z} /\left(\ell^{n}\right), \quad Z_{i}\left(G \times \mathbb{Z} /\left(\ell^{n}\right)\right)=Z_{i}(G) \times \mathbb{Z} /\left(\ell^{n}\right) .
$$

By loc. cit., Proposition 8.12, we have

$$
\begin{aligned}
& \mathrm{H}_{2}\left(Z_{i}(G) \times \mathbb{Z} /\left(\ell^{n}\right), \mathbb{Z}\right) / \mathrm{H}_{2}^{a b}\left(Z_{i}(G) \times \mathbb{Z} /(\ell)^{n}, \mathbb{Z}\right)= \\
& \mathrm{H}_{2}\left(Z_{i}(G), \mathbb{Z}\right) / \mathrm{H}_{2}^{a b}\left(Z_{i}(G), \mathbb{Z}\right) \times \mathrm{H}_{2}\left(\mathbb{Z} /\left(\ell^{n}\right), \mathbb{Z}\right) / \mathrm{H}_{2}^{a b}\left(\mathbb{Z} /\left(\ell^{n}\right), \mathbb{Z}\right)
\end{aligned}
$$

and clearly,

$$
\mathrm{H}_{2}\left(\mathbb{Z} /\left(\ell^{n}\right), \mathbb{Z}\right)=\mathrm{H}_{2}^{a b}\left(\mathbb{Z} /\left(\ell^{n}\right), \mathbb{Z}\right) .
$$

From this, the claim of the lemma follows easily.

The following proposition was proved in [FK06, Proposition 2.3.7] in the case of number fields.

Proposition 5.4. Let $Q$ be a function field of transcendence degree 1 over a finite field $\mathbb{F}$ and let $\ell$ be any prime. Then

$$
\lim _{L} \operatorname{SK}_{1}\left(\mathbb{Z}_{\ell}[\operatorname{Gal}(L / Q)]\right)=0
$$

where $L$ runs through the finite Galois extensions of $Q$ in a fixed separable closure $\bar{Q}$ of $Q$.

Proof. By the same argument as in the proof of Proposition 2.3.7 in [FK06, it suffices to prove that

for any finite extension $L$ of $Q$.

$$
\mathrm{H}^{2}\left(\operatorname{Gal}(\bar{Q} / L), \mathbb{Q}_{\ell} / \mathbb{Z}_{\ell}\right)=0
$$

If $\ell$ is different from the characteristic of $\mathbb{F}$, then the vanishing of this group can be deduced via the same argument as the analogous statement for number fields given in [Sch79, $\S 4$, Satz 1]: Let

$$
L_{\infty}=\bigcup_{n} L\left(\zeta_{\ell^{n}}\right) .
$$

Then

$$
\mathrm{H}^{2}\left(\operatorname{Gal}(\bar{Q} / L), \mathbb{Q}_{\ell} / \mathbb{Z}_{\ell}\right)=\mathrm{H}^{1}\left(\operatorname{Gal}\left(L_{\infty} / L\right), \mathrm{H}^{1}\left(\operatorname{Gal}\left(\bar{Q} / L_{\infty}\right), \mathbb{Q}_{\ell} / \mathbb{Z}_{\ell}\right)\right)
$$

and by Kummer theory,

$$
\mathrm{H}^{1}\left(\operatorname{Gal}\left(\bar{Q} / L_{\infty}\right), \mathbb{Q}_{\ell} / \mathbb{Z}_{\ell}\right)=L_{\infty}^{\times} \otimes_{\mathbb{Z}} \mathbb{Q}_{\ell} / \mathbb{Z}_{\ell}(-1) .
$$


Now

$\mathrm{H}^{1}\left(\operatorname{Gal}\left(L_{\infty} / L\right), L_{\infty}^{\times} \otimes_{\mathbb{Z}} \mathbb{Q}_{\ell} / \mathbb{Z}_{\ell}(-1)\right)=\underset{n}{\lim } \mathrm{H}^{1}\left(\operatorname{Gal}\left(L_{\infty} / L\right), L\left(\zeta_{\ell^{n}}\right)^{\times} \otimes_{\mathbb{Z}} \mathbb{Q}_{\ell} / \mathbb{Z}_{\ell}(-1)\right)$

by [NSW00, Proposition 1.5.1] and

$$
\mathrm{H}^{1}\left(\operatorname{Gal}\left(L_{\infty} / L\right), L\left(\zeta_{\ell^{n}}\right)^{\times} \otimes_{\mathbb{Z}} \mathbb{Q}_{\ell} / \mathbb{Z}_{\ell}(-1)\right)=\left(L\left(\zeta_{\ell^{n}}\right)^{\times} \otimes_{\mathbb{Z}} \mathbb{Q}_{\ell} / \mathbb{Z}_{\ell}(-1)\right)_{\operatorname{Gal}\left(L_{\infty} / L\right)}
$$

by loc. cit., Proposition 1.6.13. Since the latter group is a factor group of

$$
\left(L\left(\zeta_{\ell^{n}}\right)^{\times} \otimes_{\mathbb{Z}} \mathbb{Q}_{\ell} / \mathbb{Z}_{\ell}(-1)\right)_{\mathrm{Gal}\left(L_{\infty} / L\left(\zeta_{\ell^{n}}\right)\right)}=0,
$$

the claim is proved.

If $\ell$ is equal to the characteristic of $\mathbb{F}$, then the cohomological $\ell$-dimension of $\operatorname{Gal}(\bar{Q} / L)$ is known to be 1 [NSW00, Theorem 10.1.11.(iv)] and hence, the second cohomology group of $\mathbb{Q}_{\ell} / \mathbb{Z}_{\ell}$ vanishes for trivial reasons.

\section{L-FUnCTIONS}

Consider an adic $\operatorname{ring} \Lambda$ and let $\Lambda[[T]]$ denote the ring of power series in the indeterminate $T$ (where $T$ is assumed to commute with every element of $\Lambda$ ). The $\operatorname{ring} \Lambda[[T]]$ is still an adic ring whose Jacobson radical $\operatorname{Jac}(\Lambda[[T]])$ is generated by $\operatorname{Jac}(\Lambda)$ and $T$. In particular, we conclude from Proposition 3.5 that

$$
\mathrm{K}_{1}(\Lambda[[T]])=\lim _{n} \mathrm{~K}_{1}\left(\Lambda[[T]] / \operatorname{Jac}(\Lambda[[T]])^{n}\right)
$$

is a profinite group.

Let $\mathbb{F}$ be a finite field. We write $X_{0}$ for the set of closed points of a scheme $X$ in $\mathbf{S c h}_{\mathbb{F}}$. If $x \in X_{0}$ is a closed point, then

$$
\bar{x}=x \times_{\text {Spec } \mathbb{F}} \operatorname{Spec} \overline{\mathbb{F}}
$$

consists of finitely many points, whose number is given by the $\operatorname{degree} \operatorname{deg}(x)$ of $x$, i. e. the degree of the residue field $k(x)$ of $x$ as a field extension of $\mathbb{F}$. Let

$$
s_{x}: \bar{x} \rightarrow X
$$

denote the structure map. For any complex

$$
\mathcal{F}^{\bullet}=\left(\mathcal{F}_{I}^{\bullet}\right)_{I \in \mathfrak{I}_{\Lambda}}
$$

in $\mathbf{P D G}{ }^{\text {cont }}(X, \Lambda)$, we write

$$
\mathcal{F}_{x}^{\bullet}=\left(\Gamma\left(\bar{x}, s_{x}^{*} \mathcal{F}_{I}^{\bullet}\right)\right)_{I \in \mathfrak{I}_{\Lambda}} .
$$

This defines a Waldhausen exact functor

$$
\mathbf{P D G}^{\text {cont }}(X, \Lambda) \rightarrow \mathbf{P D G}^{\text {cont }}(\Lambda), \quad \mathcal{F}^{\bullet} \mapsto \mathcal{F}_{x}^{\bullet}
$$

Note that $\mathcal{F}_{x}^{\bullet}$ can also be written as the product over the stalks of $\mathcal{F}$ in the points of $\bar{x}$ :

$$
\mathcal{F}_{x}^{\bullet} \cong \prod_{\xi \in \bar{x}}\left(\left(\mathcal{F}_{I}^{\bullet}\right)_{\xi}\right)_{I \in \Lambda}
$$

The geometric Frobenius automorphism

$$
\mathfrak{F}_{\mathbb{F}} \in \operatorname{Gal}(\overline{\mathbb{F}} / \mathbb{F})
$$

operates on $\mathcal{F}_{x}^{\bullet}$ through its action on $\bar{x}$. Hence, it also operates on $\Psi_{\Lambda[[T]]}\left(\mathcal{F}_{x}^{\bullet}\right)$. Here,

$$
\Psi_{\Lambda[[T]]}: \mathbf{P D G}^{\text {cont }}(\Lambda) \rightarrow \mathbf{P D G}^{\text {cont }}(\Lambda[[T]])
$$

denotes the change of ring functor with respect to the $\Lambda[[T]]-\Lambda$-bimodule $\Lambda[[T]]$, as constructed in Definition 3.9. The morphism

$$
\operatorname{id}-\mathfrak{F}_{\mathbb{F}} T: \Psi_{\Lambda[[T]]}\left(\mathcal{F}_{x}^{\bullet}\right) \rightarrow \Psi_{\Lambda[[T]]}\left(\mathcal{F}_{x}^{\bullet}\right)
$$


is a natural isomorphism whose inverse is given by

$$
\sum_{n=0}^{\infty} \mathfrak{F}_{\mathbb{F}}^{n} T^{n} .
$$

Definition 6.1. The class

$$
E_{x}\left(\mathcal{F}^{\bullet}, T\right)=\left[\Psi_{\Lambda[[T]]}\left(\mathcal{F}_{x}^{\bullet}\right) \stackrel{\mathrm{id}-\mathfrak{F}_{\mathbb{E}} T}{\longrightarrow} \Psi_{\Lambda[[T]]}\left(\mathcal{F}_{x}^{\bullet}\right)\right]^{-1}
$$

in $\mathrm{K}_{1}(\Lambda[[T]])$ is called the Euler factor of $\mathcal{F}^{\bullet}$ at $x$.

One can easily verify that the Euler factor is multiplicative on exact sequences and that

$$
E_{x}\left(\mathcal{F}^{\bullet}, T\right)=E_{x}\left(\mathcal{G}^{\bullet}, T\right)
$$

if the complexes $\mathcal{F}^{\bullet}$ and $\mathcal{G}^{\bullet}$ are quasi-isomorphic. Hence, the above assignment extends to a homomorphism

$$
E_{x}(-, T): \mathrm{K}_{0}\left(\mathbf{P D G}^{\text {cont }}(X, \Lambda)\right) \rightarrow \mathrm{K}_{1}(\Lambda[[T]]) .
$$

Lemma 6.2. Let $\xi \in \bar{x}$ be a geometric point. Then

$$
E_{x}\left(\mathcal{F}^{\bullet}, T\right)=\left[\Psi_{\Lambda[[T]]}\left(\mathcal{F}_{\xi}^{\bullet}\right) \stackrel{\operatorname{id}-\widetilde{F}_{k(x)} T^{\operatorname{deg}(x)}}{\longrightarrow} \Psi_{\Lambda[[T]]}\left(\mathcal{F}_{\xi}^{\bullet}\right)\right]^{-1}
$$

Proof. The Frobenius automorphism $\mathfrak{F}_{\mathbb{F}}$ induces isomorphisms $\mathcal{F}_{\mathfrak{F}_{\mathbb{F}}^{k} \xi}^{\bullet} \cong \mathcal{F}_{\xi}^{\bullet}$ for $k=$ $1, \ldots, \operatorname{deg}(x)$. For $k=\operatorname{deg}(x)$ we have $\mathfrak{F}_{\mathbb{F}}^{k} \xi=\xi$ and the isomorphism is given by the operation of $\mathfrak{F}_{k(x)}$ on $\mathcal{F}_{\xi}^{\bullet}$. Hence, we may identify $\mathcal{F}_{x}^{\bullet}$ with the complex $\left(\mathcal{F}_{\xi}^{\bullet}\right)^{\operatorname{deg}(x)}$, on which the Frobenius $\mathfrak{F}_{\mathbb{F}}$ acts through the matrix

$$
\left(\begin{array}{ccccc}
0 & \ldots \ldots & 0 & \mathfrak{F}_{k(x)} \\
\text { id } & 0 & \ldots & \ldots & 0 \\
0 & \text { id } & 0 & \ldots & 0 \\
\vdots & \ddots & \ddots & \ddots & \vdots \\
0 & \ldots & 0 & \text { id } & 0
\end{array}\right) .
$$

Let $A$ be the automorphism of $\Psi_{\Lambda[[T]]}\left(\left(\mathcal{F}_{\xi}^{\bullet}\right)^{\operatorname{deg}(x)}\right)$ given by the matrix

$$
\left(\begin{array}{ccccc}
\operatorname{id} & 0 & \ldots \ldots & \ldots & 0 \\
\operatorname{id} T & \operatorname{id} & 0 & \ldots & 0 \\
\operatorname{id} T^{2} & \operatorname{id} T & \operatorname{id} & 0 & \vdots \\
\vdots & \ddots & \ddots & \ddots & 0 \\
\operatorname{id} T^{\operatorname{deg}(x)-1} & \ldots & \operatorname{id} T^{2} & \operatorname{id} T & \operatorname{id}
\end{array}\right)
$$

Then $A\left(\mathrm{id}-\mathfrak{F}_{\mathbb{F}} T\right)$ corresponds to the matrix

$$
\left(\begin{array}{ccccc}
\text { id } & 0 & \ldots & 0 & -\mathfrak{F}_{k(x)} T \\
0 & \text { id } & 0 & \ldots & -\mathfrak{F}_{k(x)} T^{2} \\
\vdots & & \ddots & & \vdots \\
0 & \ldots & 0 & \text { id } & -\mathfrak{F}_{k(x)} T^{\operatorname{deg}(x)-1} \\
0 & \ldots & \ldots & 0 & \left(\mathrm{id}-\mathfrak{F}_{k(x)} T^{\operatorname{deg}(x)}\right)
\end{array}\right)
$$

Moreover, we have $[A]=1$ in $\mathrm{K}_{1}(\Lambda[[T]])$. Hence,

$$
\left[\Psi_{\Lambda[[T]]}\left(\mathcal{F}_{x}^{\bullet}\right) \stackrel{\mathrm{id}-\mathfrak{F}_{\mathbb{F}} T}{\longrightarrow} \Psi_{\Lambda[[T]]}\left(\mathcal{F}_{x}^{\bullet}\right)\right]=\left[\Psi_{\Lambda[[T]]}\left(\mathcal{F}_{\xi}^{\bullet}\right) \stackrel{\mathrm{id}-\mathfrak{F}_{k(x)} T^{\operatorname{deg}(x)}}{\longrightarrow} \Psi_{\Lambda[[T]]}\left(\mathcal{F}_{\xi}^{\bullet}\right)\right]
$$

as claimed. 
Proposition 6.3. The infinite product

$$
\prod_{x \in X_{0}} E_{x}\left(\mathcal{F}^{\bullet}, T\right)
$$

converges in the profinite topology of $\mathrm{K}_{1}(\Lambda[[T]])$.

Proof. For each integer $m$, there exist only finitely many closed points $x \in X_{0}$ with $\operatorname{deg}(x)<m$. If $\operatorname{deg}(x) \geq m$, then we conclude from Lemma 6.2 that the image of $E_{x}\left(\mathcal{F}^{\bullet}, T\right)$ in $\mathrm{K}_{1}\left(\Lambda[T] /\left(T^{m}\right)\right)$ is 1 .

Definition 6.4. The $L$-function of the complex $\mathcal{F}^{\bullet}$ in $\mathbf{P D G}{ }^{\text {cont }}(X, \Lambda)$ is given by

$$
L_{\mathbb{F}}\left(\mathcal{F}^{\bullet}, T\right)=\prod_{x \in X_{0}} E_{x}\left(\mathcal{F}^{\bullet}, T\right) \in \mathrm{K}_{1}(\Lambda[[T]])
$$

Remark 6.5. If $\mathbb{F}^{\prime}$ is a subfield of $\mathbb{F}$, then Lemma 6.2 implies that

$$
L_{\mathbb{F}^{\prime}}\left(\mathcal{F}^{\bullet}, T\right)=L_{\mathbb{F}}\left(\mathcal{F}^{\bullet}, T^{\left[\mathbb{F}: \mathbb{F}^{\prime}\right]}\right) \in \mathrm{K}_{1}(\Lambda[[T]]) .
$$

Remark 6.6. If $\Lambda$ is commutative, the determinant induces an isomorphism

$$
\operatorname{det}: \mathrm{K}_{1}(\Lambda[[T]]) \rightarrow \Lambda[[T]]^{\times} .
$$

In particular, we see that the $L$-function agrees with the one defined in Del77. Fonction $\left.L \bmod \ell^{n}\right]$ in the case of commutative adic rings.

\section{The Grothendieck trace Formula}

In this section, we will prove the Grothendieck trace formula for our $L$-functions.

Definition 7.1. For a scheme $X$ in $\mathbf{S c h}_{\mathbb{F}}^{\text {sep }}$ and a complex $\mathcal{F}^{\bullet}$ in $\mathbf{P D G}{ }^{\text {cont }}(X, \Lambda)$ we let $\mathcal{L}_{\mathbb{F}}\left(\mathcal{F}^{\bullet}, T\right)$ denote the element

$$
\left[\Psi_{\Lambda[[T]]}\left(\mathrm{R}_{\mathbb{F}} \Gamma_{c}\left(\bar{X}, \mathcal{F}^{\bullet}\right)\right) \stackrel{\mathrm{id}-\mathfrak{F}_{\mathbb{F}} T}{\longrightarrow} \Psi_{\Lambda[[T]]}\left(\mathrm{R}_{\mathbb{F}} \Gamma_{c}\left(\bar{X}, \mathcal{F}^{\bullet}\right)\right)\right]^{-1}
$$

in $\mathrm{K}_{1}(\Lambda[[T]])$.

Theorem 7.2 (Grothendieck trace formula). Let $\mathbb{F}$ be a finite field of characteristic $p$ and let $\Lambda$ be an adic ring such that $p$ is invertible in $\Lambda$. Then

$$
L_{\mathbb{F}}\left(\mathcal{F}^{\bullet}, T\right)=\mathcal{L}_{\mathbb{F}}\left(\mathcal{F}^{\bullet}, T\right)
$$

for every scheme $X$ in $\mathbf{S c h}_{\mathbb{F}}^{\text {sep }}$ and every complex $\mathcal{F}^{\bullet}$ in $\mathbf{P D G}{ }^{\text {cont }}(X, \Lambda)$.

We proceed by a series of lemmas, following closely along the lines of Mil80, Chapter VI, §13].

Lemma 7.3. Let $U$ be an open subscheme of $X$ with closed complement $Z$. Theorem 7.2 is true for $X$ if it is true for $U$ and $Z$.

Proof. Write $j: U \leftrightarrow X$ and $i: Z \hookrightarrow X$ for the corresponding immersions,

$$
u: U \rightarrow \operatorname{Spec} \mathbb{F}, \quad x: X \rightarrow \operatorname{Spec} \mathbb{F}, \quad z: Z \rightarrow \operatorname{Spec} \mathbb{F}
$$

for the structure morphisms. Clearly,

$$
L\left(X, \mathcal{F}^{\bullet}\right)=L\left(U, j^{*} \mathcal{F}^{\bullet}\right) L\left(Z, i^{*} \mathcal{F}^{\bullet}\right)
$$

On the other hand, we have an exact sequence

$$
\mathrm{R} x_{!} j_{!} j^{*} \mathcal{F}^{\bullet} \longmapsto \mathrm{R} x_{!} \mathcal{F}^{\bullet} \rightarrow \mathrm{R} x_{!} i_{*} i^{*} \mathcal{F}^{\bullet}
$$

and (chains of) quasi-isomorphisms

$$
\mathrm{R} u_{!} j^{*} \mathcal{F}^{\bullet} \simeq \mathrm{R} x_{!} j_{!} j^{*} \mathcal{F}^{\bullet} \quad \mathrm{R} z_{!} i^{*} \mathcal{F}^{\bullet} \simeq \mathrm{R} x_{!} i_{*} i^{*} \mathcal{F}^{\bullet} .
$$

Hence,

$$
\left[\mathrm{R} x_{!} \mathcal{F}^{\bullet}\right]=\left[\mathrm{R} u_{!} j^{*} \mathcal{F}^{\bullet}\right]\left[\mathrm{R} z_{!} i^{*} \mathcal{F}^{\bullet}\right]
$$


in $\mathrm{K}_{0} \mathbf{P D G}^{\text {cont }}(\operatorname{Spec} \mathbb{F}, \Lambda)$. The homomorphism

$$
\begin{gathered}
\mathrm{K}_{0} \mathbf{P D G}^{\text {cont }}(\operatorname{Spec} \mathbb{F}, \Lambda) \rightarrow K_{1}(\Lambda[[T]]), \\
{\left[\mathcal{F}^{\bullet}\right] \mapsto\left[\Psi_{\Lambda[[T]]}\left(\Gamma\left(\operatorname{Spec} \overline{\mathbb{F}}, s^{*} \mathcal{F}^{\bullet}\right)\right) \stackrel{1-\mathfrak{F}_{\mathbb{F}} T}{\longrightarrow} \Psi_{\Lambda[[T]]}\left(\Gamma\left(\operatorname{Spec} \overline{\mathbb{F}}, s^{*} \mathcal{F}^{\bullet}\right)\right)\right]^{-1}}
\end{gathered}
$$

preserves this relation.

Next, we prove that the formula is compatible with change of the base field.

Lemma 7.4. Let $\mathbb{F}^{\prime}$ be a subfield of $\mathbb{F}$ and $X$ a scheme in $\mathbf{S c h}_{\mathbb{F}}^{\text {sep }}$. Then

$$
\mathcal{L}_{\mathbb{F}^{\prime}}\left(\mathcal{F}^{\bullet}, T\right)=\mathcal{L}_{\mathbb{F}}\left(\mathcal{F}^{\bullet}, T^{\left[\mathbb{F}: \mathbb{F}^{\prime}\right]}\right) .
$$

Proof. Let $r:$ Spec $\mathbb{F} \rightarrow$ Spec $\mathbb{F}^{\prime}$ be the morphism induced by the inclusion $\mathbb{F}^{\prime} \subset \mathbb{F}$ and write

$$
\begin{array}{ll}
h: X \times_{\operatorname{Spec} \mathbb{F}} \operatorname{Spec} \overline{\mathbb{F}} \rightarrow X, & h^{\prime}: X \times_{\operatorname{Spec} \mathbb{F}^{\prime}} \operatorname{Spec} \overline{\mathbb{F}} \rightarrow X \\
s: \operatorname{Spec} \overline{\mathbb{F}} \rightarrow \operatorname{Spec} \mathbb{F}, & s^{\prime}: \operatorname{Spec} \overline{\mathbb{F}} \rightarrow \operatorname{Spec} \mathbb{F}^{\prime}
\end{array}
$$

for the corresponding structure morphisms. For any $\mathcal{F}^{\bullet}$ in $\mathbf{P D G}^{\text {cont }}(X, \Lambda)$, the complexes $r_{*} \mathrm{R} h_{!} \mathcal{F}^{\bullet}, \mathrm{R} r_{!} \mathrm{R} h_{!} \mathcal{F}^{\bullet}$, and $\mathrm{R} h_{!}^{\prime} \mathcal{F}^{\bullet}$ in $\mathbf{P D G}{ }^{\text {cont }}\left(\operatorname{Spec} \mathbb{F}^{\prime}, \Lambda\right.$ ) are quasiisomorphic. Moreover, for any complex $\mathcal{G}^{\bullet}$ in $\mathbf{P D G}^{\text {cont }}(\operatorname{Spec} \mathbb{F}, \Lambda)$, the following diagram is commutative:

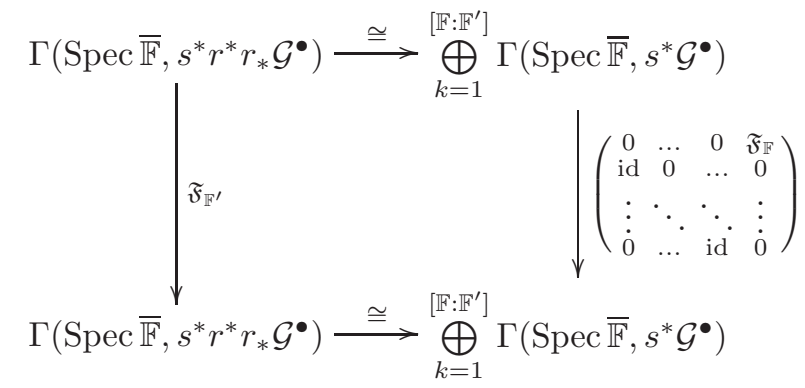

As in the proof of Lemma 6.2 one concludes

$$
\begin{array}{r}
{\left[\Psi_{\Lambda[[T]]}\left(\mathrm{R}_{\mathbb{F}^{\prime}} \Gamma_{c}\left(\bar{X}, \mathcal{F}^{\bullet}\right)\right) \stackrel{\text { id }-\mathfrak{F}_{\mathbb{F}^{\prime}} T}{\longrightarrow} \Psi_{\Lambda[[T]]}\left(\mathrm{R}_{\mathbb{F}^{\prime}} \Gamma_{c}\left(\bar{X}, \mathcal{F}^{\bullet}\right)\right)\right]=} \\
{\left[\Psi_{\Lambda[[T]]}\left(\Gamma\left(\operatorname{Spec} \overline{\mathbb{F}}, s^{*} r^{*} r_{*} \mathrm{R} h_{!} \mathcal{F}^{\bullet}\right)\right) \stackrel{\mathrm{id}-\mathfrak{F}_{\mathbb{F}^{\prime}} T}{\longrightarrow} \Psi_{\Lambda[[T]]}\left(\Gamma\left(\operatorname{Spec} \overline{\mathbb{F}}, s^{*} r^{*} r_{*} \mathrm{R} h_{!} \mathcal{F}^{\bullet}\right)\right)\right]=} \\
{\left[\Psi_{\Lambda[[T]]}\left(\Gamma\left(\operatorname{Spec} \overline{\mathbb{F}}, s^{*} \mathrm{R} h_{!} \mathcal{F}^{\bullet}\right)\right) \stackrel{\mathrm{id}-\mathfrak{F}_{\mathbb{F}} T^{\left[\mathbb{F}: \mathbb{F}^{\prime}\right]}}{\longrightarrow} \Psi_{\Lambda[[T]]}\left(\Gamma\left(\operatorname{Spec} \overline{\mathbb{F}}, s^{*} \mathrm{R} h_{!} \mathcal{F}^{\bullet}\right)\right)\right]=} \\
{\left[\Psi_{\Lambda[[T]]}\left(\mathrm{R}_{\mathbb{F}} \Gamma_{c}\left(\bar{X}, \mathcal{F}^{\bullet}\right)\right) \stackrel{\mathrm{id}-\mathfrak{F}_{\mathbb{F}} T^{\left[\mathbb{F}: \mathbb{F}^{\prime}\right]}}{\longrightarrow} \Psi_{\Lambda[[T]]}\left(\mathrm{R}_{\mathbb{F}} \Gamma_{c}\left(\bar{X}, \mathcal{F}^{\bullet}\right)\right)\right]}
\end{array}
$$

Clearly, Theorem 7.2 is true for schemes of dimension 0. Next, we consider the case that $X$ is a curve.

Lemma 7.5. The formula in Theorem 7.2 is true for any smooth and geometrically connected curve $X, \Lambda=\mathbb{Z}_{\ell}[G]$, and $\mathcal{F}^{\bullet}=\mathbb{Z}_{\ell}[G]_{X}^{\sharp}$, where $\ell$ is a prime different from the characteristic of $\mathbb{F}$ and $G$ is the Galois group of a finite Galois covering of $X$.

Proof. Let $Q$ be the function field of $X$ and let $F$ the function field of a finite Galois covering of $X$. Let $d_{F}$ denote the element

$$
d_{F}=L\left(\mathbb{Z}_{\ell}[\operatorname{Gal}(F / Q)]_{X}^{\sharp}, T\right) \mathcal{L}\left(\mathbb{Z}_{\ell}[\operatorname{Gal}(F / Q)]_{X}^{\sharp}, T\right)^{-1}
$$

in $\mathrm{K}_{1}\left(\mathbb{Z}_{\ell}[\operatorname{Gal}(F / Q)][[T]]\right)$. 
Note that $d_{F}$ does not change if we replace $X$ by an open subscheme of $X$. Hence, we may define $d_{F}$ for any finite Galois extension $F$ of $Q$. If $F^{\prime} / F$ is Galois, then $d_{F^{\prime}}$ is mapped onto $d_{F}$ under the canonical homomorphism

$$
\mathrm{K}_{1}\left(\mathbb{Z}_{\ell}\left[\operatorname{Gal}\left(F^{\prime} / Q\right)\right][[T]]\right) \rightarrow \mathrm{K}_{1}\left(\mathbb{Z}_{\ell}[\operatorname{Gal}(F / Q)][[T]]\right) .
$$

Let $L$ be a splitting field for $\mathbb{Q}_{\ell}[\operatorname{Gal}(F / Q)]$ and $M \subset L[\operatorname{Gal}(F / Q)]$ a maximal $\mathbb{Z}_{\ell^{-}}$ order. By the classical Grothendieck trace formula Del77, Fonction $L \bmod \ell^{n}$, Theorem 2.2.(a)], the image of $d_{F}$ under the homomorphism

$$
\mathrm{K}_{1}\left(\mathbb{Z}_{\ell}[\operatorname{Gal}(F / Q)][[T]]\right) \rightarrow \mathrm{K}_{1}(M[[T]]) \cong \bigoplus_{k=1}^{r} \mathcal{O}_{L}[[T]]^{\times}
$$

is trivial; hence $d_{F} \in \mathrm{SK}_{1}\left(\mathbb{Z}_{\ell}[\operatorname{Gal}(F / Q)][[T]]\right)=\mathrm{SK}_{1}\left(\mathbb{Z}_{\ell}[\operatorname{Gal}(F / Q)]\right)$. From Proposition 5.4 we conclude $d_{F}=0$.

Lemma 7.6. The formula in Theorem 7.2 is true for any scheme $X$ in $\mathbf{S c h}_{\mathbb{F}}^{\text {sep }}$ of dimension less or equal 1, any adic ring $\Lambda$ with $p \in \Lambda^{\times}$and any complex $\mathcal{F}^{\bullet}$ in $\mathbf{P D G}^{\text {cont }}(X, \Lambda)$.

Proof. By Proposition 3.5 it suffices to consider finite rings $\Lambda$. The $\ell$-Sylow subgroups of $\Lambda$ are subrings of $\Lambda$ and $\Lambda$ is equal to their direct product. Since $p$ is invertible, the $p$-Sylow subgroup is trivial. Hence, we may further assume that $\Lambda$

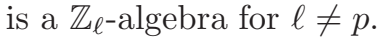

Shrinking $X$ if necessary we may assume that $X$ is smooth, irreducible curve and that $\mathcal{F}^{\bullet}$ is a strictly perfect complex of locally constant sheaves. By replacing $\mathbb{F}$ with its algebraic closure in the function field of $X$ and using Lemma 7.4, we may assume that $X$ is geometrically connected. By Lemma 4.12 and Proposition 4.11 we have

$$
\mathcal{L}\left(\mathcal{F}^{\bullet}, T\right)=\mathrm{K}_{1}\left(\Psi_{M \otimes_{\mathbb{Z}_{\ell}[G]} \mathbb{Z}_{\ell}[G][[T]]}\right)\left(\mathcal{L}\left(\mathbb{Z}_{\ell}[G]_{X}^{\sharp}, T\right)\right)
$$

for a suitable Galois group $G$ and a complex $M^{\bullet}$ in $\mathbb{Z}_{\ell}[G]^{\text {op }} \mathbf{S P P}(\Lambda)$. Likewise,

$$
L\left(\mathcal{F}^{\bullet}, T\right)=\mathrm{K}_{1}\left(\Psi_{M \otimes_{\mathbb{Z}_{\ell}[G]} \mathbb{Z}_{\ell}[G][[T]]}\right)\left(L\left(\mathbb{Z}_{\ell}[G]_{X}^{\sharp}, T\right)\right) .
$$

Now the assertion follows from Lemma 7.5 .

We complete the proof of Theorem 7.2 by induction on the dimension $d$ of $X$. By shrinking $X$ if necessary we may assume that there exists a morphism $f: X \rightarrow Y$ such that $Y$ and all fibres of $f$ have dimension less than $d$. Then Proposition 4.7.(3) and the induction hypothesis imply

$$
\mathcal{L}\left(\mathcal{F}^{\bullet}, T\right)=\mathcal{L}\left(\mathrm{R} f_{!} \mathcal{F}^{\bullet}, T\right)=L\left(\mathrm{R} f_{!} \mathcal{F}^{\bullet}, T\right) .
$$

Let now $y$ be a closed point of $Y$. Write $f_{y}: X_{y} \rightarrow X$ for the fibre over $y$. Then

$$
\begin{aligned}
E_{y}\left(\mathrm{R} f_{!} \mathcal{F}^{\bullet}, T\right) & =\left[\Psi_{\Lambda[[T]]}\left(\mathrm{R} \Gamma_{c}\left(\bar{X}_{y}, f_{y}^{*} \mathcal{F}^{\bullet}\right)\right) \stackrel{\mathrm{id}-\mathfrak{F}_{\mathbb{F}} T}{\longrightarrow} \Psi_{\Lambda[[T]]}\left(\mathrm{R} \Gamma_{c}\left(\bar{X}_{y}, f_{y}^{*} \mathcal{F}^{\bullet}\right)\right)\right]^{-1} \\
& =L\left(f_{y}^{*} \mathcal{F}^{\bullet}, T\right)
\end{aligned}
$$

by Proposition 4.7.(4) and the induction hypothesis. Since clearly

$$
L\left(\mathcal{F}^{\bullet}, T\right)=\prod_{y \in Y_{0}} L\left(f_{y}^{*} \mathcal{F}^{\bullet}, T\right),
$$

Theorem 7.2 follows.

Remark 7.7. The formula in Theorem 7.2 is also valid if $\Lambda$ is a finite field of characteristic $p$, see [Del77, Fonction $L \bmod \ell^{n}$, Theorem 2.2.(b)]. However, it does not extend to general adic $\mathbb{Z}_{p}$-algebras. We refer to loc. cit., $\S 4.5$ for a counterexample. 


\section{REFERENCES}

[AGV72] M. Artin, A. Grothendieck, and J.L. Verdier, Théorie des topos et cohomologie étale des schémas (SGA 4-3), Lecture Notes in Mathematics, no. 305, Springer, Berlin, 1972.

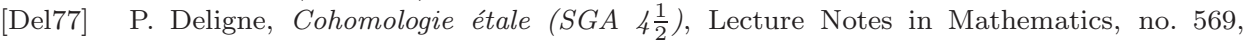
Springer, Berlin, 1977.

[FK06] T. Fukaya and K. Kato, A formulation of conjectures on p-adic zeta functions in noncommutative Iwasawa theory, Proceedings of the St. Petersburg Mathematical Society (Providence, RI), vol. XII, Amer. Math. Soc. Transl. Ser. 2, no. 219, American Math. Soc., 2006, pp. 1-85.

[Gro77] A. Grothendieck, Cohomologie $\ell$-adique et fonctions L (SGA 5), Lecture Notes in Mathematics, no. 589, Springer, Berlin, 1977.

[Lam91] T. Y. Lam, A first course in noncommutative rings, Graduate Texts in Mathematics, no. 131, Springer, Berlin, 1991.

[Mil80] J. S. Milne, Etale cohomology, Princeton Mathematical Series, no. 33, Princeton University Press, New Jersey, 1980.

[MT07] F. Muro and A. Tonks, The 1-type of a Waldhausen K-theory spectrum, Advances in Mathematics 216 (2007), no. 1, 178-211.

[MT08] _ On $K_{1}$ of a Waldhausen category, $K$-theory and noncommutative geometry, EMS Series of Congress Reports, 2008, pp. 91-116.

[NSW00] J. Neukirch, A. Schmidt, and K. Wingberg, Cohomology of number fields, Grundlehren der mathematischen Wissenschaften, no. 323, Springer Verlag, Berlin Heidelberg, 2000.

[Oli88] R. Oliver, Whitehead groups of finite groups, London Mathematical Society lecture notes series, no. 132, Cambridge University Press, Cambridge, 1988.

[Sch79] P. Schneider, Über gewisse Galoiscohomologiegruppen, Math. Z. 260 (1979), 181-205.

[Sch02] M. Schlichting, A note on K-theory and triangulated categories, Invent. Math. 150 (2002), no. 1, 111-116.

[TT90] R. W. Thomason and T. Trobaugh, Higher algebraic K-theory of schemes and derived categories, The Grothendieck Festschrift, vol. III, Progr. Math., no. 88, Birkhäuser, 1990, pp. 247-435.

[Wal85] F. Waldhausen, Algebraic K-theory of spaces, Algebraic and Geometric Topology (Berlin Heidelberg), Lecture Notes in Mathematics, no. 1126, Springer, 1985, pp. 318419.

[Wit08] M. Witte, Noncommutative Iwasawa main conjectures for varieties over finite fields, Ph.D. thesis, Universität Leipzig, 2008, available at http://www.dart-europe.eu/full.php?id=162794

Malte Witte

FAKULtät FÜR MATHEMATIK

UNIVERSITÄT REGENSBURG

UNIVERSITÄTSSTRASSE 31

D-93053 REGENSBURG

GERMANY

E-mail address: Malte.Witte@mathematik.uni-regensburg.de 